\title{
A reactive collision avoidance approach for mobile robot in dynamic environments
}

\begin{abstract}
This paper describes a novel reactive obstacle avoidance approach for mobile robot navigation in unknown and dynamic environment. This approach is developed based on the "situated-activity paradigm" and a "divide and conquer" strategy which steers the robot to move among unknown obstacles and towards a target without collision. The proposed approach entitled the Virtual Semi-Circles(VSC). The VSC approach lies in integration of 4 modules: division, evaluation, decision and motion generation. The VSC proposes a comprehensive obstacle avoidance approach for robust and reliable mobile robot navigation in cluttered, dense and complex unknown environments. The simulation result shows the feasibility and effectiveness of the proposed approach.
\end{abstract}

Keyword: Dynamic environment; Mobile robots; Obstacle avoidance; Reactive navigation 